\title{
The prevalence of programmed death ligand-1 (PD-L1) expression in non-small cell lung cancer in an unselected, consecutive population
}

\author{
Birgit Guldhammer Skov ${ }^{1} \cdot$ Sara Bird Rørvig ${ }^{1} \cdot$ Thomas Hartvig Lindkær Jensen ${ }^{1}$ Torsten Skov ${ }^{2}$
}

Received: 7 April 2019 / Revised: 7 July 2019 / Accepted: 7 July 2019 / Published online: 5 August 2019

(c) United States \& Canadian Academy of Pathology 2019

\begin{abstract}
Little is known about prevalence of PD-L1 expression in tumor cells of unselected patients with all stages of non-small cell lung cancer. The objective of this study is to assess the prevalence of PD-L1 positivity in patients with non-small cell lung cancer, to analyze the association between PD-L1 positivity and patients' clinicopathological characteristics, and to assess the use of immune-oncologic treatment in eligible patients. All non-small cell lung cancer patients diagnosed in a 10-month period in an unselected population of 1.7 million Caucasian inhabitants were evaluated with the PD-L1 IHC 22C3 pharmDx kit. A total of 819 patients were diagnosed with non-small cell lung cancer. Samples analyzable for PD-L1 expression were obtained from $97 \%$ of patients. In a multivariate analysis with cut-off at tumor proportion score $\geq 50 \%$, lower stage was associated with lower prevalence of PD-L1 positivity with an odds ratio of 0.31 for stage I vs. stage IV. A significant difference in PD-L1 expression between squamous-cell carcinoma and adenocarcinoma was observed with odds ratio for adenocarcinoma 1.8. With cut-off tumor proportion score $\geq 1 \%$, attenuated effects of the same direction were seen. For neither cut-off did type and location of material used for PD-L1 analysis, age, sex, smoking history, or performance status have statistically significant impact on the PD-L1 expression. Fifty four percent of the patients who were eligible for immune-oncologic treatment were actually treated in first-line with pembrolizumab monotherapy. In conclusion, $97 \%$ of the patients had material analyzable for PD-L1. If a patient in need of immuno-oncologic treatment has shifted stage, a negative or low positive PD-L1 test performed on a biopsy taken in a lower stage might not mirror the PD-L1 expression in the new metastatic lesion. Therefore, a re-biopsy should be considered.
\end{abstract}

\section{Introduction}

Drugs targeting the programmed cell death 1 (PD-1) receptor-ligand (PD-L1) interaction such as pembrolizumab, nivolumab, atezolizumab, and durvalumab have efficacy in advanced non-small cell lung cancer. These drugs are selective humanized monoclonal antibodies designed to block the interaction between PD-1 and its ligands and hijack a major pathway used by tumors to suppress immune control. In clinical trials with pembrolizumab [1-3], tumor expression of PD-L1 as measured

Birgit Guldhammer Skov

Bsko0020@regionh.dk

Birgit.skov@begrund.dk

1 Department of Pathology, Copenhagen University Hospital, Rigshospitalet, Denmark

2 Mørkhøj Parkalle 20, Copenhagen, Denmark by PD-L1 IHC 22C3 pharmDx assay (Agilent, Santa Clara, CA, USA), a companion diagnostic approved by the US Food and Drug Administration, has been utilized as a predictive biomarker to identify patients likely to benefit from pembrolizumab either as monotherapy in first line [1], second line [3], or combination therapy with pembrolizumab and standard chemotherapy $[4,5]$. The assessment of PD-L1 expression prior to first line treatment with pembrolizumab as monotherapy is now mandatory.

The prevalence of PD-L1 expression has largely been evaluated in clinical trial populations [1-5] or in selected populations (e.g., only histology specimens included or non-consecutive patient populations) [6,7], and/or the data has been collected from test centers rather than from consecutive and population-based series [8,9]. Little is known about prevalence of PD-L1 expression in tumor cells of unselected patients with all stages of non-small cell lung cancer, and the relationship between PD-L1 expression and baseline clinicopathological features are largely unknown. Furthermore, the evidence about the feasibility of evaluation 
of PD-L1 on cytological specimens is still not as extensive as on histological specimens and no consecutive studies of unselected patients have been reported. The extent to which patients with advanced disease who are tested PD-L1 highly positive receive first line immunotherapy is largely unknown.

We conducted a study in a strictly consecutive, unselected population from a well-defined region in the greater Copenhagen area, Denmark, covering 1.7 million Caucasian people to evaluate the prevalence of PD-L1 expression in patients with all stages of non-small cell lung cancer as assessed by the PD-L1 IHC 22C3 pharmDx kit. The PD-L1 expression was correlated to baseline clinicopathological parameters. We also assessed the proportion of eligible patients who actually were treated with immunotherapy first line.

\section{Materials and methods}

\section{Patient cohort}

This is a prospective, consecutive study including all patients with a new diagnosis of non-small cell lung cancer in a well-defined region in Denmark (RegionH) covering 1.7 million Caucasian people. The inclusion period was from 1st February 2018 to 30th November 2018. The data cutoff date for this analysis was 31st December 2018.

The completeness of the cohort is judged to be $100 \%$. Most cases of non-small cell lung cancer in the region are diagnosed at two departments of pulmonology and one department of thoracic surgery. Patients referred to other departments with unclear signs of lung cancer (typically with metastases to the liver, bone, brain or neck) were also included. All cases had the primary pathology diagnosis in our department, diagnosed by one of three pathologists (BGS, SR, TJ), or were referred to our department for PD-L1 testing. To be sure that all cases were included, a SNOMED search in the electronic pathology record system (Patobanken) was done by the end of the inclusion period, including $\mathrm{T}$ codes for lung and mediastinum, and $\mathrm{M}$ codes for malignancies. Metastases outside the thorax were identified by $\mathrm{T}$ and $\mathrm{M}$ codes combined with $Æ$ codes for origin in the lung of metastases. No cases can be signed out without SNOMED coding. Patients living outside the region were excluded by their place of residence as registered in the patient file.

The data collection criteria for this cohort were almost identical to the cohort collected to evaluate the prevalence of EGFR mutations [10].

\section{Diagnostic material}

Patients had a treatment-naive histologically and/or cytologically confirmed primary non-small cell lung cancer (all stages as classified by 8th American Joint Committee on Cancer Staging System).

Biopsy specimens and clots from cytological material were formalin fixed and paraffin embedded, and 9 slides were initially cut. Slide one and two were stained by Hematoxylin and Eosin (H\&E) and mucin stains (optionally). The following 6 unstained slides were reserved for immunohistochemistry including PD-L1 staining. The last slide was stained by H\&E to evaluate the amount of tissue available in the block after cutting. The clot material from the cytological specimens was handled in the same way as conventional histological biopsy specimens [11]. From resected specimens, slides were cut and stained by immunohistochemistry (if necessary for subclassification) and by PD-L1. Thus, all PD-L1 tests were performed on newly obtained (fresh) material and no archival specimens were included.

Diagnosis was done according to guidelines [12, 13]. In the absence of definitive conventional criteria for either squamous cell carcinoma or adenocarcinoma on histology, as well as on cytology, immunohistochemistry (primarily CK7, P40, and TTF1) was applied. The reaction was recorded as positive and negative according to normal practice.

All immunohistochemistry was done on formalin fixed and paraffin-embedded material. No immunohistochemistry (including PD-L1 staining) was performed on smears. If a patient had both histology and cytological cell block(s) available, the specimen judged as the optimal for PD-L1 test was chosen.

\section{PD-L1 staining and evaluation}

PD-L1 staining was performed at the time of the diagnosis of non-small cell lung cancer (reflex/up front testing). The assay PD-L1 IHC 22C3pharmDx, was used according to the manufacturer's instructions with the DAKO Autostainer Link-48 system. This assay is developed for detection of PD-L1 protein in formalin fixed and paraffin embedded non-small cell lung cancer tissue in combination with the EnVision FLEX visualization system.

Each PD-L1 run included positive and negative cell line slides provided by the manufacturer. Furthermore, positive controls including placenta (must exhibit strong staining of the trophoblast) and tonsil (must exhibit strong staining in portions of the crypt epithelium and weak to moderate staining of the follicular macrophages in the germinal centers) were included on every PD-L1 test slide. A negative antibody control, in which the primary antibody was replaced by negative control reagent, was done on each run.

The staining was evaluated according to instructions in the kit from the manufacturer (and thus, the same criteria were used as in the clinical trials with pembrolizumab 
[1-3]): The partial or complete membrane staining on vital malignant cells regardless of intensity was considered as a positive staining. Any cytoplasmic staining was ignored. Staining of immune cells and necrotic areas was not included in the evaluation. The percentage of stained malignant cells (tumor proportion score) was quantified on the whole section as $<1,1-49$, and $\geq 50 \%$ positive cells. The primary investigator pathologist (BGS) was trained in a Merck-sponsored program (Merck \& Co., Inc., Kenilworth, NJ, USA) to guide correct scoring and interpretation of PDL1 tumor proportion score. The two other pathologists who evaluated the PD-L1 tests (SR, TJ) were pulmonary pathologists, they were trained by BGS and had nearly 2 years of experience in reading PD-L1 test, before the initiation of the present study. In cases of uncertain PD-L1 evaluation judged by one of the pathologists, one of the other pathologists did an evaluation and a consensus value was determined.

\section{Clinicopathological data}

The following demographic, pathological, and clinical data were obtained: age and date at diagnosis, sex, type of tumor sample on which the malignant diagnosis was made, diagnosis including information regarding immunohistochemistry for diagnosis, type, and location of material for PD-L1 analysis, tumor stage (cTNM, as classified by the American Joint Committee on Cancer staging system, 8th edition), information about lung surgery or no surgery, EGFR (primarily on adenocarcinoma) as measured by Cobas ${ }^{\circledR}$ EGFR mutation Test v2 on either May-Grünwald-Giemsa stained smears or on biopsy material and ALK rearrangement (primarily on adenocarcinoma) as measured by immunohistochemistry 5A4 assay (Novocastra, Leica) if available (EGFR and ALK as up-front analyses), ECOG performance score and comorbidity (obtained by the pulmonary physician during the clinical interview prior to biopsy and registered in the electronic medical record, SP/EPIC), smoking status, including information on pack-years (never smoking was defined as less than 100 cigarettes consumed in a lifetime), previous malignant diseases (excluding nonmelanoma skin cancer, superficial bladder cancer, and in situ cervical cancer). This information was obtained from the Nationwide Pathology Databank (Patobanken), in which all pathological specimens in Denmark are registered since 1980 (a case cannot be signed out without SNOMED coding). The definition of a new non-small cell lung cancer in the study was (1) more than 3 years since the previous primary lung cancer, or (2) the new lung cancer was of different histological type than the previous lung cancer, or (3) the new lung cancer was dominated by lepidic growth.

In addition, information was obtained on whether immuno-oncologic treatment had been given for patients with tumors expressing PD-L1 $\geq 50 \%$, and, if no immuneoncologic treatment had been given, the reason for this.

Approval for the study was obtained from the local ethics committee, Rigshospitalet.

\section{Statistical analyses}

The prevalence of baseline characteristics and PD-L1 scorings were summarized using counts and proportions. Multivariate logistic regression was used to describe PD-L1 positivity related to baseline factors and type of material used for the PD-L1 analysis. Tumor tissue samples that were non-evaluable for PD-L1 analysis were described using counts and proportions.

\section{Results}

\section{Clinicopathological parameters}

In the 10-month inclusion period 819 patients were diagnosed with non-small cell lung cancer. Twenty eight patients $(3 \%)$ did not have a sample with enough material for PD-L1 analysis. Twenty of these had cytology only for diagnosis, and 20 patients were in a stage where immunooncologic treatment could potentially be an option. Seven hundred and ninety one patients were successfully tested and were included in the analyses.

Baseline characteristics of the 791 included patients are shown in Table 1 . Slightly more women than men were diagnosed with non-small cell lung cancer. The median age of the population was 71.6 years. The distribution between adenocarcinoma (68\%) and squamous-cell carcinoma $(26 \%)$ was as expected. The proportion of patients with non-small cell lung cancer, other and not otherwise specified (5\%) was acceptable. Immunohistochemical markers, including CK7, TTF1, P40, and CK5, were used to aid $78 \%$ of the diagnoses. "Other" non-small cell lung cancer included 3 patients with sarcomatoid carcinoma, 1 patient with large cell carcinoma, and 5 patients with combined small cell carcinoma ( 3 with component of adenocarcinoma and 2 with component of squamous-cell carcinoma).

The proportion of patients who had cytology only was $28 \%$. All except one of these had a cell block (clot) that could be used for ancillary studies including immunohistochemistry. Fifteen out of the 791 patients were re-biopsied to get enough material for PD-L1 analysis (data not shown).

For $83 \%$ of the patients whose PD-L1 was analyzed on a histologic sample, the material was from a biopsy (core needle, bronchial biopsy or transbronchial biopsy), $17 \%$ were from resected specimens. Ninety one percent of the histological material used for PD-L1 analyses was from the primary tumor. In contrast, less than $1 / 3$ of the PD-L1 
Table 1 Baseline characteristic for the included 791 with enough material for PD-L1 analysis

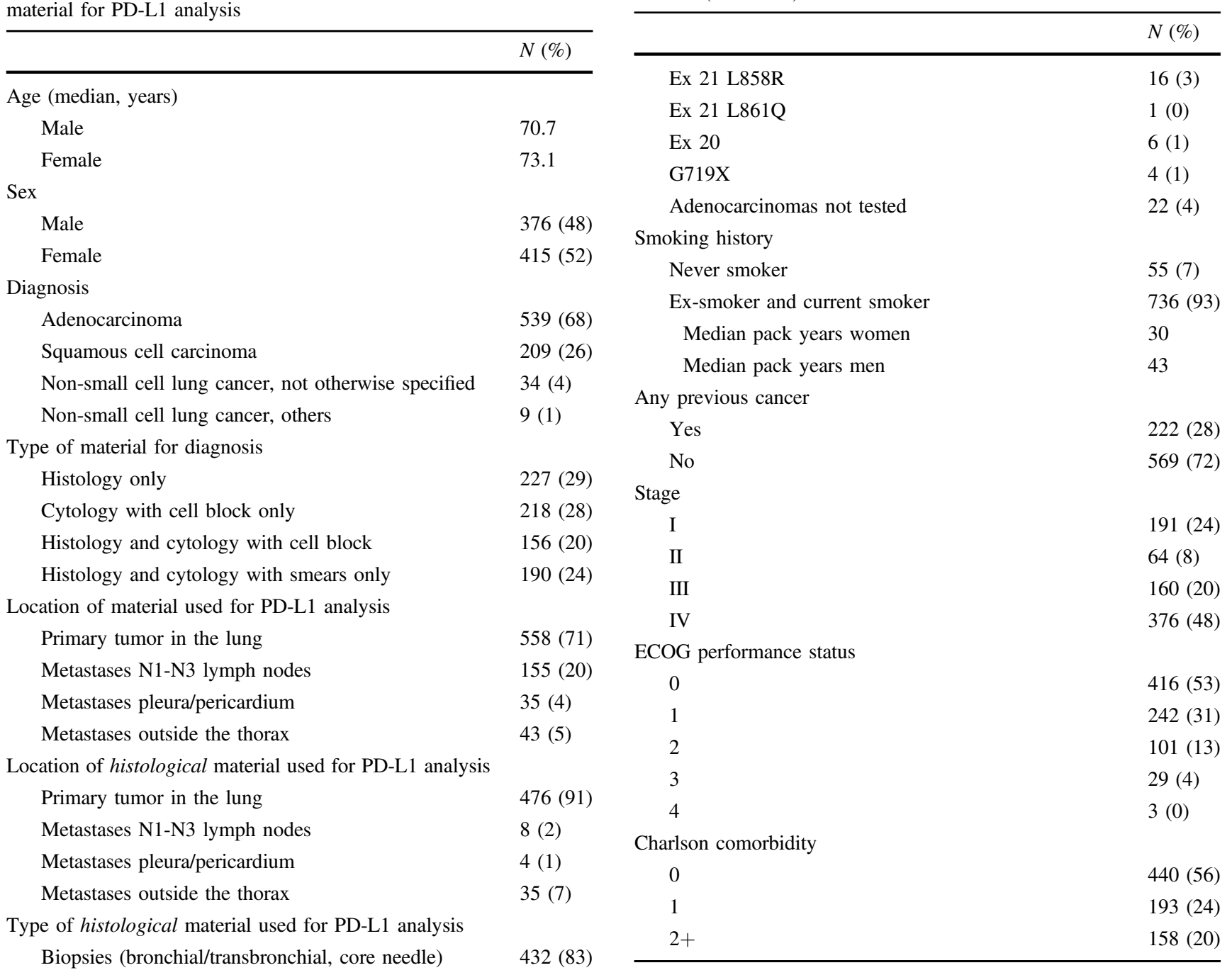

Table 1 (continued)
$91(17)$

$31(12)$

$8(3)$

$168(63)$

ALK status

Positive

Negative

Adenocarcinomas not tested

EGFR mutation status

Wild type

Del 19 $542(98)$ analyses performed on cytology was from the primary tumor. About $2 / 3$ of the cytological material used for the PD-L1 analyses was obtained by endoscopic ultrasound and endobronchial ultrasound. Twelve percent of the cytological material was from pleura/pericardial effusion.

Alk status was evaluated primarily in adenocarcinoma and most of the adenocarcinoma patients were tested (98\%). In the whole population 11 patients $(2 \%)$ had an ALK translocation, 10 of these had also material for PD-L1 analysis.

EGFR status was evaluated primarily in adenocarcinoma and most adenocarcinoma patients were tested (95\%). The EGFR mutation rate was $8.3 \%$. Activating mutations were seen in $7.2 \%$ of the tested patients.

The proportion of never smokers was $7 \%$. In the smoking population, $83 \%$ had $>15$ packyears. Twenty eight percent of all patients had a previous cancer. Fifty six patients previously had lung cancer (data not shown). In almost all patients, staging included PET-CT. Forty 
eight percent were diagnosed in stage IV, $20 \%$ were diagnosed in locally advanced stage. Five patients could not be given a detailed cTNM stage because they were Tx or Nx and M0.

Performance status and comorbidity was judged by the pulmonary physician at the initial contact with the hospital. Seventeen percent were performance status 2 or higher. Forty four percent of included patients had comorbidity.

\section{PD-L1 expression}

Four hundred and ninety nine patients $(63 \%$ (95\% confidence interval $60-67 \%$ )) had PD-L1 $\geq 1 \%$ positive cells. Two hundred and forty patients $(30 \%$ (95\% confidence interval 27-34\%)) had PD-L1 $\geq 50 \%$ positive cells.

Table 2 show the relation between PD-L1 expression and baseline characteristics. Since many of these variables are correlated, the associations should be interpreted with caution and no statistical tests are provided. A multivariate analysis is included below which disentangles these associations.

Slightly more women than men had PD-L1 $\geq 50 \%$. Slightly more men than women had PD-L1 between $1 \%$ and $49 \%$. Among cases of adenocarcinoma and non-small cell lung cancer, other and not otherwise specified, more had PD-L1 $\geq 50 \%$ compared to squamous-cell carcinoma. More cases of squamous-cell carcinoma had PD-L1 between $1 \%$ and $49 \%$ compared to adenocarcinoma and non-small cell lung cancer, other and not otherwise specified. TTF1 positive adenocarcinoma had $\geq 50 \%$ PD-L1 expression in $38 \%$ vs. $16 \%$ in TTF1 negatives (data not shown). PD-L1 expression was higher in higher TNM stages than in lower stages. The higher expression is seen especially in the $\geq 50 \%$ column and the lower expression in the $<1 \%$ column, whereas the intermediate column is largely unchanged over stage.

Regarding ALK translocation, the numbers in each category are too small for interpretation. Cases with activating EGFR mutations express PD-L1 to a lower extent than the EGFR wild type cases, but the numbers are very small.

More tumors tested on cytology expressed PD-L1 $\geq 50 \%$ than tumors tested on histology (36\% vs. 27\%). No consistent pattern of PD-L1 expression was seen in cytology vs. histology in stages III and IV. In stages I and II there was a trend towards higher PD-L1 in cytology than in histology, but the numbers are small.

More tumors tested from smokers expressed PD-L1 $\geq 50 \%$ than tumors tested from never smokers.

Fifty four percent of the patients with a PD-L1 score of at least $50 \%$ and stage IIIA, IIIB/IIIC, and stage IV were treated in first-line with pembrolizumab. More than $90 \%$ were in stage IV. Among the reasons for not giving
Table 2 PD-L1 staining by baseline characteristics

\begin{tabular}{|c|c|c|c|c|}
\hline & \multicolumn{4}{|c|}{ PD-L1 staining } \\
\hline & $<1 \% N(\%)$ & $\begin{array}{l}1-49 \% \\
N(\%)\end{array}$ & $\begin{array}{l}\geq 50 \% \\
N(\%)\end{array}$ & Total $N(\%)$ \\
\hline \multicolumn{5}{|l|}{ Sex } \\
\hline Male & $137(36)$ & $132(35)$ & $107(28)$ & $376(100)$ \\
\hline Female & $155(37)$ & $127(31)$ & $133(32)$ & $415(100)$ \\
\hline \multicolumn{5}{|l|}{ ALK status* } \\
\hline Positive & $6(60)$ & $2(20)$ & $2(20)$ & $10(100)$ \\
\hline Negative & $204(38)$ & $158(29)$ & $180(33)$ & $542(100)$ \\
\hline \multicolumn{5}{|l|}{ EGFR mutation status** } \\
\hline $\begin{array}{l}\text { Activating } \\
\text { mutation }\end{array}$ & $17(47)$ & $13(36)$ & $6(17)$ & $36(100)$ \\
\hline $\begin{array}{l}\text { Ex } 20 \text { non- } \\
\text { activating } \\
\text { mutation }\end{array}$ & $4(67)$ & 0 & $2(33)$ & $6(100)$ \\
\hline Wild type & $172(36)$ & $137(30)$ & $165(35)$ & $474(100)$ \\
\hline \multicolumn{5}{|c|}{ Material used for PD-L1 analysis } \\
\hline Cytology & $88(33)$ & $83(31)$ & $97(36)$ & $268(100)$ \\
\hline Histology & $204(39)$ & $176(34)$ & $143(27)$ & $523(100)$ \\
\hline \multicolumn{5}{|c|}{ Location of used material for PD-L1 analysis } \\
\hline $\begin{array}{l}\text { Primary tumor in } \\
\text { the lung }\end{array}$ & $217(39)$ & $196(35)$ & $145(26)$ & $558(100)$ \\
\hline Metastasis & $75(32)$ & $63(27)$ & $95(41)$ & $233(100)$ \\
\hline \multicolumn{5}{|l|}{ Stage } \\
\hline I & $87(46)$ & $74(39)$ & $30(16)$ & $191(100)$ \\
\hline II & $26(41)$ & $22(34)$ & $16(25)$ & $64(100)$ \\
\hline III & $56(35)$ & $59(37)$ & $45(28)$ & $160(100)$ \\
\hline IV & $123(33)$ & $104(27)$ & $149(40)$ & $376(100)$ \\
\hline \multicolumn{5}{|l|}{ Diagnosis } \\
\hline Adenocarcinomas & $202(37)$ & $160(30)$ & $177(33) 44$ & $539(100)$ \\
\hline $\begin{array}{l}\text { Squamous cell } \\
\text { carcinomas }\end{array}$ & $75(36)$ & $90(43)$ & (21) & $209(100)$ \\
\hline $\begin{array}{l}\text { Non-small cell } \\
\text { lung cancer, not } \\
\text { otherwise } \\
\text { specified }\end{array}$ & $10(29)$ & $8(24)$ & $16(47)$ & $34(100)$ \\
\hline $\begin{array}{l}\text { Non-small cell } \\
\text { lung cancer, other }\end{array}$ & $5(56)$ & $1(11)$ & $3(33)$ & $9(100)$ \\
\hline
\end{tabular}

*Four patients had no material for PD-L1 analysis and for ALK test

**There are 44 EGFR mutated in the full analysis set but only 42 in the PD-L1 analysis set. Twenty two patients in the PD-L1 analysis set had no material for EGFR analysis

immuno-oncologic treatment were performance status 2 or higher, brain metastases, and irradiation often in combination with prednisolone treatment. More patients with no comorbidity $(N=71(70 \%))$ received immuno-oncologic therapy than patients with 1 or more comorbidities $(N=23$ $(32 \%))$ and the difference was clearly significant. Although comorbidity was likely to have influenced for example performance status, it was not given as the primary reason for not treating with immuno-oncologic therapy.

The multivariate analysis showed that at tumor proportion score $\geq 50 \%$, cTNM stage and diagnosis were statistically significant at the $5 \%$ level while controlling for age, sex, type of material used, location of material used, smoking, and performance status which were not statistically significant (Table 3 ). There was less PD-L1 positivity 
Table 3 Multivariate analysis of PD-L1 positivity defined as $\geq 50$ and $\geq 1 \%$ positive tumor cells

\begin{tabular}{|c|c|c|c|c|}
\hline & \multicolumn{2}{|l|}{$\geq 50 \%$ positive } & \multicolumn{2}{|l|}{$\geq 1 \%$ positive } \\
\hline & $\begin{array}{l}\text { Odds ratio }(95 \% \\
\text { confidence interval) }\end{array}$ & $P$-value & $\begin{array}{l}\text { Odds ratio }(95 \% \\
\text { confidence interval) }\end{array}$ & $P$-value \\
\hline \multicolumn{5}{|l|}{ Diagnosis } \\
\hline $\begin{array}{l}\text { Adenocarcinoma vs. squamous-cell } \\
\text { carcinoma }\end{array}$ & $1.81(1.20-2.73)$ & 0.003 & $0.92(0.64-1.31)$ & 0.66 \\
\hline $\begin{array}{l}\text { Non-small cell lung cancer, not } \\
\text { otherwise specified vs. squamous- } \\
\text { cell carcinoma }\end{array}$ & $3.02(1.39-6.58)$ & & $1.29(0.57-2.88)$ & \\
\hline \multicolumn{5}{|l|}{ Stage } \\
\hline I vs. IV & $0.31(0.19-0.51)$ & & $0.59(0.39-0.89)$ & \\
\hline II vs. IV & $0.58(0.30-1.12)$ & $<.0001$ & $0.74(0.41-1.34)$ & 0.08 \\
\hline III vs. IV & $0.67(0.44-1.03)$ & & $0.92(0.61-1.39)$ & \\
\hline
\end{tabular}

The models included diagnosis, stage, age, sex, type of material used, location of material used, smoking, and performance status in lower stages with an odds ratio of 0.31 for stage I vs. stage IV. Adenocarcinoma had odds ratio around 1.8, and non-small cell lung cancer, not otherwise specified had odds ratio 3. For tumor proportion score $\geq 1 \%$, the effects of diagnosis and stage were attenuated and not statistically significant in the full model shown in Table 3. When only stage and diagnosis were included in the model, stage had a $p$-value of 0.03 and diagnosis had a $p$-value of 0.75 .

For neither cut-off, did type and location of material used for PD-L1 analysis, age, sex, smoking history, or performance status have statistically significant impact on the PDL1 expression.

\section{Discussion}

This is the largest study of PD-L1 prevalence in an unselected, consecutive cohort of non-small cell lung cancer Caucasian patients. Samples that could be analyzed for PDL1 expression were obtained from $97 \%$ of all lung cancer patients in the cohort.

With cut-off at tumor proportion score $\geq 50 \%$, lower stage was associated with lower prevalence of PD-L1 positivity with an odds ratio of 0.31 for stage I vs. stage IV. A significant difference in PD-L1 expression between squamouscell carcinoma and adenocarcinoma was observed with odds ratio for adenocarcinoma around 1.8. With cut-off tumor proportion score $\geq 1 \%$, attenuated effects of the same direction were seen. For neither cut-off, did type and location of material used for PD-L1 analysis, age, sex, smoking status, and performance status have statistically significant impact on the PD-L1 expression level. In other words, our data indicate that the PD-L1 analysis can be performed on the primary tumor or on the metastasis, on a histological biopsy or a cytological cell block. The clinical implication seems to be that if a patient in need of immuno-oncologic treatment has shifted stage, a negative or low positive PD-L1 test performed on a biopsy taken in a lower stage might not mirror the PD-L1 expression in the new metastatic lesion. Therefore, a re-biopsy should be considered.

Using PD-L1 IHC 22C3 pharmDx, Wang et al. [8] showed that for tumor proportion score $\geq 50 \%$, a statistically significant 2.3-fold higher prevalence of PD-L1 positivity was seen in patients with metastatic disease compared to early/locally advanced disease. Evans et al. [14] found that tumor samples taken from the primary tumor in the lung showed significantly lower rates of PDL1 positivity (using PD-L1 IHC 22C3 pharmDx) than samples from pleura and lymph nodes. In two large cohorts of resected patients, higher PD-L1 positivity was detected in higher stages with PD-L1 IHC 28-8 pharmDx [15] or PD-L1 IHC 22C3 pharmDx and PD-L1 SP263 [7]. A trend toward higher PD-L1 expression in higher stages (PD-L1 IHC 22C3 pharmDx) [6] or no correlation to stage (PD-L1 antibody E1L3N) have also been noted [16]. In the later using PD-L1 $\geq 50 \%$ a discrepancy between primary tumor and N2 nodes was observed in 3 out of 32 cases $(9.4 \%)$. In one of these cases the primary tumor was positive and the N2 metastasis was negative. Heterogeneity of the PD-L1 expression may be the explanation for this discrepancy.

In an updated analysis of the Keynote-010 trial Herbst et al. [17] compared the outcome in patients based on PDL1 test performed on archival vs. newly tumor samples. Regarding overall survival, a newly acquired biopsy was somewhat more predictive compared to an archival biopsy (for tumor proportion score $\geq 50 \%$, the overall survival hazard ratios were 0.64 (95\% confidence interval: 0.45 , $0.91)$ and 0.40 (95\% confidence interval: $0.28,0.56)$ for archival and newly collected samples, respectively). No information was provided about the tumor stage of the material used for PD-L1 analysis. 
In the present study more than $25 \%$ of the patients had cytology only for diagnosis and thus also for PD-L1 testing. In the clinical trials that have led to approval of immunooncologic therapy including pembrolizumab, only histologic specimens were allowed and for this reason the assay has been approved for histological specimens only. In the literature it is debated whether the result of a PD-L1 test preformed on cytology is accurate. If PD-L1 testing on cytology were to be deemed unacceptable, a large proportion of non-small cell lung cancer patients would need either a new (histologic) biopsy (with risk of complications) or would be deprived of immuno-oncologic therapy. We reported in 2017 that a high agreement $\left(R^{2}=0.87-0.89\right)$ was found for PD-L1 IHC 22C3 pharmDX and PD-L1 IHC 28-8 pharmDX whether applied to histologic or cytologic cell blocks with an overall agreement between $85 \%$ and 95\% [11]. Cases with disagreement of PD-L1 expression between cytology and histology were largely cases with a heterogeneous expression of PD-L1 in the histological material. Since then more studies have come to the same conclusion [18-20]. Thus, on the analytical level PD-L1 testing on cytology seems trustable, although there is no consensus about the clinically acceptable degree of statistical agreement.

We demonstrated that more PD-L1 was observed in cytological specimens compared to histological specimens. The multivariate analysis indicated that this was due to cytological specimens being sampled from higher stages than histological specimens. In two recent studies the same difference in PD-L1 expression between cytology and histology was reported. Wang et al. [8] demonstrated that for surgical resections, small biopsies, and cytology the percentages of specimens with tumor proportion score $\geq 50$ were $29 \%, 36 \%$, and $42 \%$, respectively. The numbers reported by Evans et al. were $22.5 \%, 29 \%$, and $39.2 \%$, respectively [14]. Both studies used PD-L1 IHC 22C3pharmDX.

One way to examine if PD-L1 testing on cytology is trustable on the same level as PD-L1 testing on histology is to evaluate response rate and survival for patients tested on either material. Only very sparse data on this topic is available. Torous et al. [21] showed that in 20 patients with PD-L1 tumor proportion score $\geq 50 \%, 11$ were tested on cytology and the clinical outcome for these patients was comparable with patients tested on histology. In a study of 38 patients no significant difference regarding progressive disease and disease control after immuno-oncologic treatment first line for tumor proportion score $\geq 50 \%$ was observed between patients PD-L1 tested on small biopsies $(N=25)$ and cytology $(N=13)$ [22].

In the majority of studies in which the agreement of PDL1 expression on cytology and histology has been evaluated, the PD-L1 22C3 pharmDX on cell block material has been used [11, 18-20, 23]. Given suitable cell block material (with at least a H\&E stained slides and also likely immunohistochemistry) stained by PD-L1 $22 \mathrm{C} 3$ pharmDX used according to the manufacturer's instructions and an experienced pathologist, the PD-L1 test on cell blocks seems to be applicable. Fewer publications have evaluated other PD-L1 assays on cytology [24, 25], and for these antibodies more data is needed. The impact of preanalytical factors including fixative must also be further evaluated and the same is true for PD-L1 staining on liquid-based cytology and smeared material [26].

We found significant differences in PD-L1 expression between adenocarcinoma and squamous-cell carcinoma, the former having the highest expression. Other studies have reported similar findings [27, 28], whereas some have demonstrated higher expression in squamous-cell carcinoma [6] or no difference [14, 29]. Our study included nearly 800 consecutive patients and was controlled for confounders including sex, smoking habits, type and location of material for PD-L1 analysis and stage.

In our institution, (small) biopsies and cytological cell blocks are initially cut in serial sections with blank slides for ancillary studies including PD-L1 testing. This procedure is most likely the reason for the very high proportion of patients having material for ancillary studies including PDL1 (and ALK and EGFR analyses).

The percentages of EGFR mutations and the ALK positive rate were identical to a similar population some years ago $[10,30]$. The reproducibility of these numbers indicate that they mirror the true rates of these genetic abnormalities. Due to small numbers, no conclusion could be drawn about the association between PD-L1 expression and EGFR and ALK status.

Fifty four percent (93 patients) of the patients with a PDL1 score of at least $50 \%$ and stage IIIB/IIIC and stage IV were treated in first-line with pembrolizumab. Forty two of these patients were PD-L1 tested on cytological cell block material. A follow up of the outcome of all these patients is planned.

In conclusion, in this large, consecutive study of PD-L1 expression in all stages of non-small cell lung cancer, it was demonstrated that $97 \%$ of the patients had material for PDL1 analysis. Stage was the most important predictor of PDL1 expression with higher stages having higher prevalence of positive tumors. For tumor proportion score $\geq 50 \%$ more adenocarcinoma than squamous-cell carcinoma were PD-L1 positive. Type and location of material used for PD-L1 analysis, age, sex, smoking history, or performance status had no statistically significant impact on the PD-L1 expression.

Acknowledgements We thank Mette Pøhl, Jon Andersen, Lotte Engell-Nørregård, Jens Benn Sørensen, Lars Drivsholm, Amal 
Durakovic, Klaus Richter Larsen, and Jesper Ravn for providing some of the clinical data from the medical records.

\section{Compliance with ethical standards}

Conflict of interest BGS has received honoraria (consultation or lectures) fees from MSD, AstraZeneca, BMS and Roche. The remaining authors declare that they have no conflict of interest.

Publisher's note: Springer Nature remains neutral with regard to jurisdictional claims in published maps and institutional affiliations.

\section{References}

1. Reck M, Rodriguez-Abreu D, Robinson AG, Hui R, Csoszi T, Fulop A, et al. Pembrolizumab versus chemotherapy for PD-L1positive non-small-cell lung cancer. N Engl J Med. 2016;375: 1823-33.

2. Garon EB, Rizvi NA, Hui R, Leighl N, Balmanoukian AS, Eder JP, et al. Pembrolizumab for the treatment of non-small-cell lung cancer. N Engl J Med. 2015;372:2018-28.

3. Herbst RS, Baas P, Kim DW, Felip E, Perez-Gracia JL, Han JY, et al. Pembrolizumab versus docetaxel for previously treated, PD-L1-positive, advanced non-small-cell lung cancer (KEYNOTE-010): a randomised controlled trial. Lancet. 2016; 387:1540-50.

4. Gandhi L, Rodriguez-Abreu D, Gadgeel S, Esteban E, Felip E, De Angelis F, et al. Pembrolizumab plus chemotherapy in metastatic non-small-cell lung cancer. N Engl J Med. 2018;378:2078-92.

5. Paz-Ares L, Luft A, Vicente D, Tafreshi A, Gumus M, Mazieres J, et al. Pembrolizumab plus chemotherapy for squamous non-smallcell lung cancer. N Engl J Med. 2018;379:2040-51.

6. Ye L, Leslie C, Jacques A, Mesbah Ardakani N, Amanuel B, Millward M. Programmed death ligand-1 expression in non-small cell lung cancer in a Western Australian population and correlation with clinicopathologic features. Mod Pathol. 2018;32:524.

7. Song P, Guo L, Li W, Zhang F, Ying J, Gao S. Clinicopathologic correlation with expression of PD-L1 on both tumor cells and tumor-infiltrating immune cells in patients with non-small cell lung cancer. J Immunother. 2019;42:23-8.

8. Wang H, Agulnik J, Kasymjanova G, Wang A, Jimenez P, Cohen $\mathrm{V}$, et al. Cytology cell blocks are suitable for immunohistochemical testing for PD-L1 in lung cancer. Ann Oncol. 2018;29: 1417-22.

9. Evans M, O'Sullivan B, Hughes F, Mullis T, Smith M, Trim N, et al. The clinicopathological and molecular associations of PD-L1 expression in non-small cell lung cancer: analysis of a series of 10,005 cases tested with the 22C3 Assay. Pathol Oncol Res. 2018 Sep 17; [Epub ahead of print].

10. Skov BG, Hogdall E, Clementsen P, Krasnik M, Larsen KR, Sorensen JB, et al. The prevalence of EGFR mutations in nonsmall cell lung cancer in an unselected Caucasian population. APMIS. 2015;123:108-15.

11. Skov BG, Skov T. Paired comparison of PD-L1 expression on cytologic and histologic specimens from malignancies in the lung assessed with PD-L1 IHC 28-8pharmDx and PD-L1 IHC 22C3pharmDx. Appl Immunohistochem Mol Morphol. 2017; 25:453-9.

12. Travis WD, Brambilla E, Nicholson AG, Yatabe Y, Austin JH, Beasley MB, et al. The 2015 World Health Organization Classification of Lung Tumors: impact of genetic, clinical and radiologic advances since the 2004 classification. J Thorac Oncol. 2015; 10:1243-60.
13. Yatabe Y, Dacic S, Borczuk AC, Warth A, Russell PA, Lantuejoul $\mathrm{S}$, et al. Best practices recommendations for diagnostic immunohistochemistry in lung cancer. J Thorac Oncol. 2019;14:377-407.

14. Evans M, O'Sullivan B, Smith M, Taniere P. Predictive markers for anti-PD-1/PD-L1 therapy in non-small cell lung cancer-where are we? Transl Lung Cancer Res. 2018;7:682-90.

15. Kerr KM, Thunnissen E, Dafni U, Finn SP, Bubendorf L, Soltermann A, et al. A retrospective cohort study of PD-L1 prevalence, molecular associations and clinical outcomes in patients with NSCLC: results from the European Thoracic Oncology Platform (ETOP) Lungscape Project. Lung Cancer. 2019;131:95-103.

16. Uruga H, Bozkurtlar E, Huynh TG, Muzikansky A, Goto Y, Gomez-Caraballo M, et al. Programmed cell death ligand (PD-L1) expression in stage II and III lung adenocarcinomas and nodal metastases. J Thorac Oncol. 2017;12:458-66.

17. Herbst RS, Baas P, Perez-Gracia JL, Felip E, Kim DW, Han JY, et al. Use of archival versus newly collected tumor samples for assessing PD-L1 expression and overall survival: an updated analysis of KEYNOTE-010 trial. Ann Oncol. 2019;30:281-9.

18. Ilie M, Juco J, Huang L, Hofman V, Khambata-Ford S, Hofman P. Use of the $22 \mathrm{C} 3$ anti-programmed death-ligand 1 antibody to determine programmed death-ligand 1 expression in cytology samples obtained from non-small cell lung cancer patients. Cancer Cytopathol. 2018;126:264-74.

19. Heymann JJ, Bulman WA, Swinarski D, Pagan CA, Crapanzano JP, Haghighi M, et al. PD-L1 expression in non-small cell lung carcinoma: Comparison among cytology, small biopsy, and surgical resection specimens. Cancer Cytopathol. 2017;125:896-907.

20. Noll B, Wang WL, Gong Y, Zhao J, Kalhor N, Prieto V, et al. Programmed death ligand 1 testing in non-small cell lung carcinoma cytology cell block and aspirate smear preparations. Cancer Cytopathol. 2018;126:342-52.

21. Torous VF, Rangachari D, Gallant BP, Shea M, Costa DB, VanderLaan PA. PD-L1 testing using the clone 22C3 pharmDx kit for selection of patients with non-small cell lung cancer to receive immune checkpoint inhibitor therapy: are cytology cell blocks a viable option? J Am Soc Cytopathol. 2018;7:133-41.

22. Guo K, Kasymjanova G, Wang H, Sakr L, Small D, Cohen V, et al. Comparison of clinical response to checkpoint inhibitors in advanced NSCLC with high PD-L1 expression tested on cytology versus biopsy samples. J Am Soc Cyctopathol. 2018;13(P1): 04-16.

23. Hernandez A, Brandler TC, Zhou F, Moreira AL, Schatz-Siemers N, Simsir A. Assessment of programmed death-ligand 1 (PD-L1) immunohistochemical expression on cytology specimens in nonsmall cell lung carcinoma. Am J Clin Pathol. 2019;151:403-15.

24. Capizzi E, Ricci C, Giunchi F, Zagnoni S, Ceccarelli C, Gomez BUA, et al. Validation of the immunohistochemical expression of programmed death ligand 1 (PD-L1) on cytological smears in advanced non small cell lung cancer. Lung Cancer. 2018;126: 9-14.

25. Russell-Goldman E, Kravets S, Dahlberg SE, Sholl LM, Vivero M. Cytologic-histologic correlation of programmed death-ligand 1 immunohistochemistry in lung carcinomas. Cancer Cytopathol. 2018;126:253-63.

26. Jain D, Sukumar S, Mohan A, Iyer VK. Programmed death-ligand 1 immunoexpression in matched biopsy and liquid-based cytology samples of advanced stage non-small cell lung carcinomas. Cytopathology. 2018;29:550-7.

27. Kitazono S, Fujiwara Y, Tsuta K, Utsumi H, Kanda S, Horinouchi $\mathrm{H}$, et al. Reliability of small biopsy samples compared with resected specimens for the determination of programmed deathligand 1 expression in non-small-cell lung cancer. Clin Lung Cancer. 2015;16:385-90. 
28. Ota K, Azuma K, Kawahara A, Hattori S, Iwama E, Tanizaki J, et al. Induction of PD-L1 expression by the EML4-ALK oncoprotein and downstream signaling pathways in non-small cell lung cancer. Clin Cancer Res. 2015;21:4014-21.

29. Ilie M, Long-Mira E, Bence C, Butori C, Lassalle S, Bouhlel L, et al. Comparative study of the PD-L1 status between surgically resected specimens and matched biopsies of NSCLC patients reveal major discordances: a potential issue for anti-PD-L1 therapeutic strategies. Ann Oncol. 2016;27:147-53.

30. Skov BG, Clementsen P, Larsen KR, Sorensen JB, Mellemgaard A. The prevalence of ALK rearrangement in pulmonary adenocarcinomas in an unselected Caucasian population from a defined catchment area: impact of smoking. Histopathology. 2017; 70:889-95. 